\title{
ABOUT NECESSITY OF DEVELOPMENT A COMMON METHOD OF TRAINING OF STUDENTS IN NATURAL SCIENCE TO SOLVE ECOLOGICAL AND ENVIRONMENTAL PROBLEMS
}

\author{
William Hogland \\ Vasilii Rud' \\ Anastasia Stepanova \\ Linnaeus University, Sweden \\ Saint-Petersburg State Polytechnic University, Russia
}

\begin{abstract}
The problem of worsening of ecological situation on the Earth and in the Nordic and the Baltic countries requires a detailed search to find solutions ecological and environmental problems. It is obviously that their decision may occur on various fronts: reducing greenhouse gas emissions, increasing the share of energy plants, new water treatment technologies, management of municipal solid waste, etc.

However, not only technology, but also the skills of engineers, scientists and support staff underlie of each of these approaches. Therefore it's important to make a decision in training of highly qualified personnel to develop and manage new technologies comes out on top.

The Nordic and the Baltic Sea region consists of 10 states, each of which has its own system of training specialists in the field of ecology and environmental. One of the aims is not only to analyze the set of natural-scientific disciplines that are studied by the future specialists in this area and their volume, but also to create (develop) a single, brand new for the Nordic and Baltic region, the method of training, based on an analysis of existing systems.

This approach can be implemented on the basis of the traditional cooperation in this area between the St. Petersburg State Polytechnic University ( SPbSPU) (Russia) and the University of Linnaeus ( Sweden), involving other universities, producing companies and the management authorities of cities of the Baltic and northern regions and the creation of an extensive network on the subject.
\end{abstract}

\section{KEYWORDS}

Baltic see region, Skills, Environmental, St. Petersburg State Polytechnic University, SPbSPU, University of Linnaeus, Natural sciences.

\section{INTRODUCTION}

The main goal of study is to form students' holistic science ideology. 
Programs relying on knowledge that students received at schools should be obliged to provide further phased intensification of modern representations in physics, chemistry and ecology. All stages of studying nature sciences must be interconnected that will give an engineer a great opportunity to decide problem questions of environmental protection.

How we do it in Saint-Petersburg State Polytechnic University? Let's represent natural sciences programs analysis.

\section{PROJECT OVERVIEW}

Physics is a basis for natural sciences study. Physics training program for students in Saint-Petersburg State Polytechnic University is distributed as follows: lectures - 4 hours per week, practice - 2 hours per week and laboratory - 2 hours per week. The course covers following sections of physics: mechanics, electricity, magnetism, optics, quantum optics, atom, nuclear, elements of quantum mechanics, molecular physics and thermodynamics.

Mechanics section includes the following topics: kinematics of material point and rigid body, the main kinematic characteristics of motion, the transformation of velocity and acceleration during the transition to a different reference frame. Dynamics of material point: Newton's laws, the conservation and change of momentum and center of mass principles, noninertial frame. Mechanical energy and work, the conservation of energy principle, elastic and inelastic collisions. The principle of the conservation of angular momentum. Moment of force and angular momentum relative to a point and an axis, rigid body rotational motion dynamics, moment of inertia, motion of a rigid body.

Electricity and magnetism sections include: Coulomb's law, the electric field strength and electric potential, the principle of superposition, Gauss's law and its applications, dielectrics and Gauss's law for the electric displacement, the energy of the electric field, Biot-Savart law, flux of magnetic field, magnetic, law of electromagnetic induction, Maxwell's equations.

Optics and quantum optics sections begin with a chapter on geometric optics, which contains laws of geometrical optics, Fermat's principle and a description of simple optical systems and tasks related to a phenomenon of total internal reflection, plane and spherical waves interference, temporary and spatial coherence. One of the chapters in this section is devoted to diffraction: the Fresnel's diffraction and Fraunhofer's diffraction by a slit, circular aperture and a diffraction grating. The use of a diffraction grating as a spectral device, polarization, birefringence, dispersion of light and others are studied in these sections.

In conclusion of this section there are such elements of quantum optics as laws of thermal radiation and Planck's formula, energy and momentum of photons, photoelectric effect, Compton's effect, pressure of light. 
Last two sections - atom and core and elements of statistic physics and thermodynamics are studied by students themselves.

There is a course of labs where students are free to decide some practical problems of phenomena and effects study.

There are 10 labs that are directly relevant to the theoretical (lecture) course. These labs can be done on computerized lab machines. Descriptions of laboratory works are prepared and being in the laboratory.

Chemistry training program is distributed as follows: lectures -2 hours per week, labs 1 hour per week. The course covers following sections of chemistry: basic concepts and laws of chemistry, atomic structure, periodic law and Mendeleev's periodic table of chemical elements, matter chemical bond, structure and properties, energetics and direction of chemical processes, chemical kinetics, catalysis, chemical equilibrium, solutions, redox reactions, electrochemical processes, metals and alloys.

Basic concepts and laws of chemistry section includes next aspects: the chemistry place in the nature sciences, subject and tasks of chemistry, chemical production and environmental protection, the atomic and molecular doctrine, chemical elements, simple and complex matter, individual substances and mixtures, ideal and real gases, Avagadro's law and its consequences, the equation of state of an ideal gas, partial gas pressure.

Atomic structure and periodic law and periodic table of chemical elements sections include the information about structure of an atom (Rutherford's model), isotopes, radioactivity ( $\alpha$-decay, $\beta$-decay), wave-particle duality (Planck's formula, de Broglie's equation), the uncertainty principle, quantum and mechanic theory of atomic structure, quantum numbers in Schrödinger's equation, structure of atomic electron shells (Hund's rule), periodic law and periodic system of chemical elements, energy characteristics of an atom: ionization energy, electron affinity and electronegativity.

Ecology training program is distributed as follows: lectures - 2 hours per week and laboratory - 1 hour per week. Basic fundamental concepts of modern ecology are in the program. These concepts main goal is to form holistic representations of biosphere, man's place in it, problems associated with the interaction between society and nature in the era of technological civilization.

The course task is to develop noosphere thinking and skills to evaluate human activity and results of their work from the position of the preserving natural and cultural environment and the ability to direct their professional activities to the conservation of the biosphere as a habitat for humans.

The ecology course includes next sections: doctrine fundamentals of biosphere, evolution and natural balance of ecosystems, human impact on the environment; modern ecological crisis and the role of science in its overcoming, the problem of 
protecting the human environment, the international legal environment; problems of human survival, international cooperation in environmental protection.

It is real to assume that the relationship between time and depth of individual issues of each nature sciences to the needs of future specialists in the field of environmental engineering protection may have a different level. It is likely that the same relationship between time and depth can be established between both separate tasks of one section and different sections in general.

There is a logical question: is it possible to make an ideal course of natural sciences that includes everything that is necessary only for one environment protection engineers' degree?

After all we can compare the range of issues that are raised to an engineer because of the present situation and level of the used mechanisms, technologies and methodologies and the phenomena and laws that are in the so-called basic levels of natural sciences study?

We think that this problem and some research of 10 countries of the Baltic region experience will give us a possibility to make the first step towards the optimization of time and depth in teaching separate sections of natural sciences for environment protection engineers.

\section{ACKNOWLEDGEMENTS}

We thanks for Linnaeus University and for IVA for supporting. 